\title{
One-pot synthesis of new series 3,4,5-trisubstituted-dihydroisoxazoline derivatives via 1,3-dipolar cycloaddition of nitrile oxides with chalcones
}

\author{
RAAD KASIM YHYA*, K M LOKANATHA RAI and EBRAHEEM ABDU MUSAD \\ Department of studies in Chemistry, University of Mysore, Manasagangotri, Mysore 570 006, India \\ e-mail: raad_1965@yahoo.com
}

MS received 28 November 2012; revised 22 April 2013; accepted 17 May 2013

\begin{abstract}
We have synthesized a series of novel isoxazolines via 1,3-dipolar cycloaddition reaction. Aromatic aldoximes undergo oxidative-dehydrogenation with chloramine-T to give nitrile oxides, which were reacted with chalcones to afford of 3,4,5-trisubstituted 4,5-dihydroisoxazolines in a good yield.
\end{abstract}

Keywords. Isoxazoline; 1,3-dipolar cycloaddition; aromatic aldoximes; chalcones.

\section{Introduction}

Heterocyclic compounds have wide range of application in synthetic organic chemistry. Among them five-membered heterocycles, isoxazolines are of considerable interest due to their versatile application in pharmaceutical and agrochemical agents. Isoxazoline derivatives have been reported to possess antidiabetic, ${ }^{1}$ antiinfluenza virus, ${ }^{2}$ antifungal, ${ }^{3}$ glycoprotein $\mathrm{IIb} / \mathrm{IIIa}$ receptor antagonists, ${ }^{4}$ antiHIV, ${ }^{5}$ spermicidal and antiHIV, $^{6}$ analgesic and antiinflammatory, ${ }^{7}$ and $\beta$-adrenergic receptor antagonist, ${ }^{8}$ antitumour, ${ }^{9}$ antistress ${ }^{10}$ and anticancer properties. ${ }^{11}$ Isoxazolines also act as an important building block for the synthesis of biologically active molecules. ${ }^{12}$ In fact, Valdecoxib is an isoxazoline derivative, now widely used in the market as an antiinflammatory drug. ${ }^{13}$

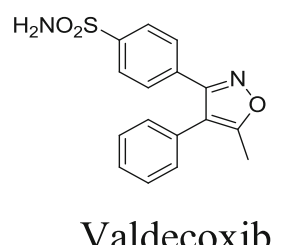

1,3-Dipolar cycloadditions are powerful methods for building a variety of five-membered heterocycles in a convergent manner from simpler molecules which are otherwise accessible only through a difficult synthetic exercise. Cycloaddition of nitrile oxide to olefinic

*For correspondence compounds are of synthetic interest, since the resulting isoxazolines are versatile intermediates for the synthesis of bifunctional compounds. ${ }^{14}$ Nitrile oxides can be generated by dehydrogenation of aryl aldoximes with mercuric acetate, ${ }^{15}$ manganese dioxide, ${ }^{16}$ tertbutyl hypochlorite, ${ }^{17}$ chloramine-T. ${ }^{18}$ In 1989 Hassner and Rai have reported the synthesis of isoxazolines via the formation of nitrile oxides from crossponding oxime and the subsequent reaction with olefins and suggested a mechanism for the reaction. ${ }^{19}$ Recently, Ganoker et al. used chloramine-T for the generation and cycloaddition of $\alpha$-nitrosoolefin and $\alpha$-azoalkenes from ketoximes and ketone hydrazones, ${ }^{20}$ respectively. Yongjia et al. synthesized 3,4,5-trisubstituted isoxazoles from 3,4,5-trisubstituted isoxazolines. ${ }^{21}$ With this background, herein we report the cycloaddition reaction of different aldoximes with various chalcones.

\section{Experimental}

\subsection{General}

The IR spectra (in $\mathrm{KBr}$ pellets) were recorded on JASCO FT/IR-460/113257 spectometer (Japan) in the wave number range of $4000-400 \mathrm{~cm}^{-1}$.

Elemental analyses were carried out on an Elementor vairo-EL instrument. ${ }^{1} \mathrm{H}-\mathrm{NMR}(400 \mathrm{MHz})$ and ${ }^{13} \mathrm{C}$ NMR $(100 \mathrm{MHz})$ spectra were measured on $\mathrm{CDCl}_{3}$ and tetramethylsilane is used as internal reference. The mass analysis was performed on HP-5989A LC/MS spectrometer. The solvents and reagents were used without further purification. 
2.2 General procedure for synthesis of chalcones $(\mathbf{l a}-\mathbf{g})^{22}$

Acetophenone $(0.01 \mathrm{~mol})$ and aromatic aldehyde $(0.01 \mathrm{~mol})$ were dissolved in $(5 \mathrm{ml}, 95 \%)$ ethanol. $0.5 \mathrm{ml}, 60 \%$ solution of sodium hydroxide was added slowly and stirred the mixture until it solidifies and then adding $10 \mathrm{ml}$ of ice water. The solid product was filtered, dried and purified from ethanol.

\subsection{General procedure for synthesis of aromatic aldoximes $(2 \boldsymbol{a}-\boldsymbol{c})^{23}$}

Aromatic aldehyde (benzaldehyde or 3-methoxybenzaldehydeor 3,4-dimethoxybenzaldehyde) $(0.1 \mathrm{~mol})$ in $15 \mathrm{ml}$ ethanol was added to a solution of hydroxylamine hydrochloride $(0.14 \mathrm{~mol})$ and sodium acetate $(0.14 \mathrm{~mol})$ the mixture was heated at $80-90^{\circ} \mathrm{C}$ for $1 \mathrm{~h}$ and then left to cool to room temperature. The precipitate was collected and purified by crystallization from ethanol to give compound (2a-g).

\subsection{General procedure for synthesis of isoxazoline $(3 a a-3 g c)$}

A mixture of chalcone $(5 \mathrm{mmol})$, oxime $(5 \mathrm{mmol})$ and chloramine- $\mathrm{T}(5.2 \mathrm{mmol})$ in ethanol $(20 \mathrm{ml})$ was refluxed on a water-bath. After $2 \mathrm{~h}$, the reaction was monitored by TLC. After completion, the mixture was cooled to room temperature. Sodium chloride formed was filtered off and washed with ethanol $(15 \mathrm{ml})$. Filtrate and washing were combined and the solvent was evaporated in vacuum. The residue was extracted with ether $(25 \mathrm{ml})$, the extract was washed successively with water $(2 \times 15 \mathrm{ml}), 10 \% \mathrm{NaOH}$ $(2 \times 15 \mathrm{ml})$, and saturated brine solution $(10 \mathrm{ml})$. The organic layer was dried over anhydrous sodium sulphate. The crude product was filtered and purified by column chromatography on silica gel using chloroform-methanol to give the corresponding pure product.

2.4a Isoxazoline 3aa: Obtained as brown oil from chalcone 1a and oxime 2a, IR (neat, $\mathrm{cm}^{-1}$ ): 3180 , 3074, 2924, 1678, 1616, 1234: 1H-NMR (400 MHz, $\left.\mathrm{CDCl}_{3}\right): \delta 3.40\left(\mathrm{~d}, 8.2 \mathrm{~Hz}, 1 \mathrm{H}, \mathrm{C}_{4}\right), 5.46(\mathrm{~d}, 8.2 \mathrm{~Hz}$, $\left.1 \mathrm{H}, \mathrm{C}_{5}\right), 7.27-8.08(\mathrm{~m}, 15 \mathrm{H}, \mathrm{ArH}), 10.00$ (br, $1 \mathrm{H}$, $\mathrm{NH}) .{ }^{13} \mathrm{C}-\mathrm{NMR}\left(100 \mathrm{~Hz}, \mathrm{CDCl}_{3}, \mathrm{rt}\right): 47.13,83.81$, $109.01,126.76,127.18,127.86,128.60,128.92$, $129.48,129.95,131.53,132.25,138.17,141.53$, 152.19, 155.28, 196.57, Anal. Calcd. for $\mathrm{C}_{22} \mathrm{H}_{17} \mathrm{NO}_{2}$ : C
80.71, H 5.23, N 4.28, found C 80.90, H 5.20, N 4.39. LC-MS m/z: $328.13(\mathrm{M}+1)^{+}$.

2.4b Isoxazoline 3ab: Obtained as yellow oil from chalcone 1a and oxim 2b, IR (neat, $\mathrm{cm}^{-1}$ ): 3210, 3045, 2953, 1680, 1612, 1245, 1173: ${ }^{1} \mathrm{H}-\mathrm{NMR}(400 \mathrm{MHz}$, $\left.\mathrm{CDCl}_{3}, \mathrm{rt}\right): \delta 3.76\left(\mathrm{~s}, 3 \mathrm{H}, \mathrm{OCH}_{3}\right), 3.49(\mathrm{~d}, 8.2 \mathrm{~Hz}, 1 \mathrm{H}$, $\left.\mathrm{C}_{4}\right), 5.20\left(\mathrm{~d}, 8.2 \mathrm{~Hz}, 1 \mathrm{H}, \mathrm{C}_{5}\right), 6.80-8.08(\mathrm{~m}, 14 \mathrm{H}, \mathrm{ArH})$, 9.90 (s, $1 \mathrm{H}, \mathrm{NH}) .{ }^{13} \mathrm{C}-\mathrm{NMR}\left(100 \mathrm{~Hz}, \mathrm{CDCl}_{3}, \mathrm{rt}\right): 44.31$, 57.15, 83.74, 109.23, 119.96, 122.81, 126.65, 127.52, $128.60,128.94,134.50,138.00,141.74,153.42$, 155.60, 160.57, 197.62. Anal. Calcd. for $\mathrm{C}_{23} \mathrm{H}_{19} \mathrm{NO}_{3}: \mathrm{C}$ 77.29, H 5.36, N 3.92, found C 77.50, H 5.49, N 3.99. LC-MS m/z: $358.14(\mathrm{M}+1)^{+}$.

2.4c Isoxazoline 3ac: Obtained as pale yellow oil from chalcone 1a and oxime $\mathbf{2 c}$, IR (neat, $\mathrm{cm}^{-1}$ ): 3196, 3071, 2973, 1660, 1610, 1241, 1192, ${ }^{1} \mathrm{H}-\mathrm{NMR}$ $\left(400 \mathrm{MHz}, \mathrm{CDCl}_{3}\right): \delta 3.77\left(\mathrm{~s}, 6 \mathrm{H}, \mathrm{OCH}_{3}\right), 3.52(\mathrm{~d}$, $\left.8.4 \mathrm{~Hz}, 1 \mathrm{H}, \mathrm{C}_{4}\right), 5.39\left(\mathrm{~d}, 8.4 \mathrm{~Hz}, 1 \mathrm{H}, \mathrm{C}_{5}\right), 6.65-8.04$ $(\mathrm{m}, 13 \mathrm{H}, \mathrm{ArH}), 9.75$ (br, $1 \mathrm{H}, \mathrm{NH}) .{ }^{13} \mathrm{C}-\mathrm{NMR}(100 \mathrm{~Hz}$, $\left.\mathrm{CDCl}_{3}, \mathrm{rt}\right): 45.49,57.13,84.38,108.35,115.46,121.76$, $124.45,127.11,127.62,128.40,128.63,131.74$, $136.27,139.91,146.65,150.12,153.50,155.70$, 199.02. Anal. Calcd. for $\mathrm{C}_{24} \mathrm{H}_{21} \mathrm{NO}_{4}$ : C 74.40, H 5.46, N 3.62, found C 74.50, H 5.20, N 3.39. LC-MS m/z: $388.15(\mathrm{M}+1)^{+}$.

2.4d Isoxazoline 3ba: Obtained as yellow oil from chalcone $\mathbf{1 b}$ and oxime $\mathbf{2 a}$, IR (neat, $\mathrm{cm}^{-1}$ ): 3185,3035 , 2971, 1671, 1618, 1241: ${ }^{1} \mathrm{H}-\mathrm{NMR}\left(400 \mathrm{MHz}, \mathrm{CDCl}_{3}\right.$ ), rt): $\delta 2.40\left(\mathrm{~s}, 3 \mathrm{H}, \mathrm{CH}_{3}\right), 3.51\left(\mathrm{~d}, 8.2 \mathrm{~Hz}, 1 \mathrm{H}, \mathrm{C}_{4}\right), 5.60$ $\left(\mathrm{d}, 8.2 \mathrm{~Hz}, 1 \mathrm{H}, \mathrm{C}_{5}\right), 7.22-8.05(\mathrm{~d}, 14 \mathrm{H}, \mathrm{ArH}), 9.81$ (s, $1 \mathrm{H}, \mathrm{NH}) .{ }^{13} \mathrm{C}-\mathrm{NMR}\left(100 \mathrm{MHz}, \mathrm{CDCl}_{3}, \mathrm{rt}\right): 21.19$, 43.51, 86.30, 105.96, 126.33, 127.52, 128.33, 128.72, $129.35,131.21,131.93,133.14,135.65,136.14$, 136.81, 153.43, 155.18, 199.03. Anal. CHN: Calcd. For $\mathrm{C}_{23} \mathrm{H}_{19} \mathrm{NO}_{2}$ : C 80.92, H 5.61, N 4.10, found C 80.76, H 5.87, N 4.01. LC-MS m/z: $342.14(\mathrm{M}+1)^{+}$.

2.4e Isoxazoline $\mathbf{3} \boldsymbol{b} \boldsymbol{b}$ : Obtained as yellow oil from chalcone $\mathbf{1 b}$ and oxime $\mathbf{2 b}$, IR (neat, $\mathrm{cm}^{-1}$ ): 3205, 3038, 2946, 1675, 1618, 1237: ${ }^{1} \mathrm{H}-\mathrm{NMR}\left(400 \mathrm{MHz}, \mathrm{CDCl}_{3}\right.$, rt): $\delta 2.39\left(\mathrm{~s}, 3 \mathrm{H}, \mathrm{CH}_{3}\right), 3.50\left(\mathrm{~d}, 8.2 \mathrm{~Hz}, 1 \mathrm{H}, \mathrm{C}_{4}\right), 3.81$ $\left(\mathrm{s}, 3 \mathrm{H}, \mathrm{OCH}_{3}\right), 5.60\left(\mathrm{~d}, 8.2 \mathrm{~Hz}, 1 \mathrm{H}, \mathrm{C}_{5}\right), 6.80-8.06(\mathrm{~m}$, $13 \mathrm{H}, \mathrm{ArH}), 9.50$ (br, $1 \mathrm{H}, \mathrm{NH}) .{ }^{13} \mathrm{C}-\mathrm{NMR}(100 \mathrm{MHz}$, $\left.\mathrm{CDCl}_{3}, \mathrm{rt}\right):$ 19.75, 45.61, 58.13, 85.14, 106.42, 121.1, $122.62,125.77,126.83,128.61,130.14,137.30$, 138.10, 140.23, 153.34, 156.43, 165.15, 195.67. Anal. Calcd. for $\mathrm{C}_{24} \mathrm{H}_{21} \mathrm{NO}_{3}$ : C 77.61, $\mathrm{H}$ 5.70, N 3.77 found $\mathrm{C}$ 77.56, H 5.99, N 3.43. LC-MS m/z: $372.15(\mathrm{M}+1)^{+}$. 
2.4f Isoxazoline 3bc: Obtained as pale brown oil from chalcone $\mathbf{1 b}$ and oxime $\mathbf{2 c}$, IR (neat, $\mathrm{cm}^{-1}$ ): 3218, 3036, 2978, 1694, 1628, 1244, 1178: ${ }^{1} \mathrm{H}-\mathrm{NMR}$ $\left(400 \mathrm{MHz}, \mathrm{CDCl}_{3}, \mathrm{rt}\right): \delta 2.43\left(\mathrm{~s}, 3 \mathrm{H}, \mathrm{CH}_{3}\right), 3.55(\mathrm{~d}$, $\left.8.4 \mathrm{~Hz}, 1 \mathrm{H}, \mathrm{C}_{4}\right), 3.90\left(\mathrm{~s}, 6 \mathrm{H}, \mathrm{OCH}_{3}\right), 5.46(\mathrm{~d}, 8.4 \mathrm{~Hz}$, $\left.1 \mathrm{H}, \mathrm{C}_{5}\right) 7.26-7.82(\mathrm{~m}, 12 \mathrm{H}, \mathrm{ArH}), 10.02(\mathrm{~s}, 1 \mathrm{H}, \mathrm{NH})$. ${ }^{13} \mathrm{C}-\mathrm{NMR}\left(100 \mathrm{MHz}, \mathrm{CDCl}_{3}, \mathrm{rt}\right): 22.12,44.45,57.21$, 87.13, 107.66, 114.73, 122.50, 125.61, 126.84, 128.11, $128.65,132.64,135.88,136.16,139.50,142.19$, 145.11, 153.13, 155.60, 196.82. Anal. CHN: Calcd. for $\mathrm{C}_{25} \mathrm{H}_{23} \mathrm{NO}_{4}$ : C 74.79, $\mathrm{H}$ 5.77, N 3.49, found C 74.69, $\mathrm{H}$ 5.92, N 3.33. LC-MS m/z: $402.16(\mathrm{M}+1)^{+}$.

2.4g Isoxazoline 3ca: Obtained as yellow oil from chalcone 1c and oxime 2a, IR (neat, $\mathrm{cm}^{-1}$ ) 3205, 3061, 2927, 1681, 1622, 1253: ${ }^{1} \mathrm{H}-\mathrm{NMR}\left(400 \mathrm{MHz}, \mathrm{CDCl}_{3}\right.$, $\mathrm{rt}): \delta 3.61\left(\mathrm{~d}, 8.2 \mathrm{~Hz}, 1 \mathrm{H}, \mathrm{C}_{4}\right), 5.14(\mathrm{~d}, 8.2 \mathrm{~Hz}, 1 \mathrm{H}$, $\left.\mathrm{C}_{5}\right), 7.26-8.02(\mathrm{~m}, 14 \mathrm{H}, \mathrm{ArH}), 10.20(\mathrm{~s}, 1 \mathrm{H}, \mathrm{NH})$. ${ }^{13} \mathrm{C}-\mathrm{NMR}\left(100 \mathrm{MHz}, \mathrm{CDCl}_{3}, \mathrm{rt}\right): 48.07,86.42,107.52$, $125.54,127.85,128.17,128.38,128.92,129.33$, 130.19, 132.29, 134.04, 137.56, 138.01, 139.9, 141.10, 153.88, 156.96, 198.20. Anal. Calcd. for $\mathrm{C}_{22} \mathrm{H}_{16} \mathrm{ClNO}_{2}$ : C 73.03, H 4.46, N 3.87, found C 73.20, H 4.33, N 3.95. LC-MS m/z: $363.09(\mathrm{M}+2)^{+}$.

2.4h Isoxazoline $3 \boldsymbol{c b}$ : Obtained as pale brown oil from chalcone $\mathbf{1 c}$ and oxime $\mathbf{2 b}$, IR (neat, $\mathrm{cm}^{-1}$ ): 3183, 3096, 2954, 1678, 1620, 1240, 1197: ${ }^{1} \mathrm{H}-\mathrm{NMR}$ $\left(400 \mathrm{MHz}, \mathrm{CDCl}_{3}, \mathrm{rt}\right): \delta 3.52\left(\mathrm{~d}, 8.2 \mathrm{~Hz}, 1 \mathrm{H}, \mathrm{C}_{4}\right), 3.86$ (s, 3H, $\left.\mathrm{OCH}_{3}\right), 5.60\left(\mathrm{~d}, 8.2 \mathrm{~Hz}, 1 \mathrm{H}, \mathrm{C}_{5}\right), 6.80-8.01$ (m, 13H, ArH), 9.50 (s, 1H, NH). ${ }^{13} \mathrm{C}-\mathrm{NMR}(100 \mathrm{MHz}$, $\left.\mathrm{CDCl}_{3}, \mathrm{rt}\right): 48.12,57.37,86.95,109.21,124.15,128.40$, $128.57,129.10,129.59,131.30,132.13,136.78$, 140.66, 152.40, 155.90, 160.18, 198.50. Anal. Calcd. for $\mathrm{C}_{23} \mathrm{H}_{18} \mathrm{ClNO}_{3}$ : C 70.50, $\mathrm{H} 4.63, \mathrm{~N} 3.57$, found $\mathrm{C}$ 70.39, H 4.81, N 3.46. LC-MS m/z: $393.10(\mathrm{M}+2)^{+}$.

2.4i Isoxazoline 3cc: Obtained as brown oil from chalcone 1c and oxime 2c, IR (neat, $\mathrm{cm}^{-1}$ ): 3192, 3078, 2951, 1680, 1618, 1240, 1186: ${ }^{1} \mathrm{H}-\mathrm{NMR}$ $\left(400 \mathrm{MHz}, \mathrm{CDCl}_{3}, \mathrm{rt}\right): \delta 3.74\left(\mathrm{~d}, 8.4 \mathrm{~Hz}, 1 \mathrm{H}, \mathrm{C}_{4}\right)$, $3.83\left(\mathrm{~s}, 6 \mathrm{H}, \mathrm{OCH}_{3}\right), 5.62\left(\mathrm{~d}, 8.4 \mathrm{~Hz}, 1 \mathrm{H}, \mathrm{C}_{5}\right), 6.93-$ $8.02(\mathrm{~m}, 12 \mathrm{H}, \mathrm{ArH}), 10.14(\mathrm{~s}, 1 \mathrm{H}, \mathrm{NH}) \cdot{ }^{13} \mathrm{C}-\mathrm{NMR}$ $\left(100 \mathrm{MHz}, \mathrm{CDCl}_{3}, \mathrm{rt}\right): 45.81,54.73,83.45,108.83$, $117.36,124.82,126.60,128.14,128.61,130.20$, $132.22,134.50,135.61,137.31,139.18,148.02$, 149.16, 150.34, 156.63, 199,13. Anal. Calcd. for $\mathrm{C}_{24} \mathrm{H}_{20} \mathrm{ClNO}_{4}$ : C 68.33, H 4.78, N 3.32, found C 68.13, H 4.80, N 3.13. LC-MS m/z: $423.11(\mathrm{M}+2)^{+}$.
2.4 $\mathrm{j}$ Isoxazoline 3da: Obtained as pale brown oil from chalcone 1d and oxime 2a, IR (neat, $\mathrm{cm}^{-1}$ ): 3211, 3081, 2988, 1671, 1610, 1235: ${ }^{1} \mathrm{H}-\mathrm{NMR}$ $\left(400 \mathrm{MHz}, \mathrm{CDCl}_{3}, \mathrm{rt}\right): \delta 3.40\left(\mathrm{~d}, 8.4 \mathrm{~Hz}, 1 \mathrm{H}, \mathrm{C}_{4}\right), 5.71$ $\left(\mathrm{d}, 8.4 \mathrm{~Hz}, 1 \mathrm{H}, \mathrm{C}_{5}\right), 7.26-8.23(\mathrm{~m}, 14 \mathrm{H}, \mathrm{ArH}), 10.10$ (br, $1 \mathrm{H}, \mathrm{NH}) .{ }^{13} \mathrm{C}-\mathrm{NMR}\left(100 \mathrm{MHz}, \mathrm{CDCl}_{3}, \mathrm{rt}\right): 48.63$, 88.72, 110.10, 122.51, 127.73, 128.15, 128.93, 129.26, $129.88,131.65,133.17,139.08,145.73,152.51$, 158.16, 193.49. Anal. Calcd. for $\mathrm{C}_{22} \mathrm{H}_{16} \mathrm{~N}_{2} \mathrm{O}_{4}$ : C 70.96, $\mathrm{H} 4.33$, N 7.52, found C 70.79, H 4.53, N 7.41. LC-MS $\mathrm{m} / \mathrm{z}: 373.11(\mathrm{M}+1)^{+}$.

2.4k Isoxazoline $3 \boldsymbol{d} \boldsymbol{b}$ : Obtained as pale brown oil from chalcone $\mathbf{1 d}$ and oxime $\mathbf{2 b}$, IR (neat, $\mathrm{cm}^{-1}$ ): 3178 , 3051, 2986, 1691, 1609, 1241: ${ }^{1} \mathrm{H}-\mathrm{NMR}(400 \mathrm{MHz}$, $\left.\mathrm{CDCl}_{3}, \mathrm{rt}\right): \delta 3.41\left(\mathrm{~d}, 8.2 \mathrm{~Hz}, 1 \mathrm{H}, \mathrm{C}_{4}\right), 3.76(\mathrm{~s}, 3 \mathrm{H}$, $\left.\mathrm{OCH}_{3}\right), 5.47\left(\mathrm{~d}, 8.2 \mathrm{~Hz}, 1 \mathrm{H}, \mathrm{C}_{5}\right), 6.81-7.99(\mathrm{~m}, 13 \mathrm{H}$, $\mathrm{ArH}), 11.21(\mathrm{~s}, \quad 1 \mathrm{H}, \mathrm{NH}) .{ }^{13} \mathrm{C}-\mathrm{NMR} \quad(100 \mathrm{MHz}$, $\left.\mathrm{CDCl}_{3}, \mathrm{rt}\right): 47.12,55.73,82.67,110.31,121.83,128.71$, $129.20,130.40,131.32,139.07,141.42,148.13$, 152.40, 158.20, 160.03, 198.11. Anal. Calcd. for $\mathrm{C}_{23} \mathrm{H}_{18} \mathrm{~N}_{2} \mathrm{O}_{5}$ : C 68.65, H 4.51, N 6.96, found C 68.85, H 4.31, N 6.98. LC-MS m/z: $403.12(\mathrm{M}+1)^{+}$.

2.41 Isoxazoline 3dc: Obtained as brown oil from chalcone 1d and oxime 2c, IR (neat, $\mathrm{cm}^{-1}$ ): 3183, 3062, 2945, 1684, 1621, 1245, 1190: ${ }^{1} \mathrm{H}-\mathrm{NMR}(400 \mathrm{MHz}$, $\left.\mathrm{CDCl}_{3}, \mathrm{rt}\right): \delta 3.43\left(\mathrm{~d}, 8.4 \mathrm{~Hz}, 1 \mathrm{H}, \mathrm{C}_{4}\right), 3.89(\mathrm{~s}, 6 \mathrm{H}$, $\left.\mathrm{OCH}_{3}\right), 5.85\left(\mathrm{~d}, 8.4 \mathrm{~Hz}, 1 \mathrm{H}, \mathrm{C}_{5}\right), 6.88-8.31(\mathrm{~m}, 12 \mathrm{H}$, $\mathrm{ArH}), 10.13$ (s, $1 \mathrm{H}, \mathrm{NH}) .{ }^{13} \mathrm{C}-\mathrm{NMR}\left(100 \mathrm{MHz}, \mathrm{CDCl}_{3}\right.$, rt): 45.88, 57.21, 86.20, 110.11, 112.83, 121.15, 122.94, $125.36,127.82,128.01,133.75,135.19,145.07$, 147.44, 149.65, 150.50, 152.70, 156.06, 193.76. Anal. Calcd. for $\mathrm{C}_{24} \mathrm{H}_{20} \mathrm{~N}_{2} \mathrm{O}_{6}: \mathrm{C}$ 66.66, $\mathrm{H}$ 4.66, $\mathrm{N}$ 6.48, found $\mathrm{C}$ 66.87, H 4.55, N 6.69. LC-MS m/z: 433.13 $(\mathrm{M}+1)^{+}$.

2.4m Isoxazoline 3ea: Obtained as brown oil from chalcone 1e and oxime 2a, IR (neat, $\mathrm{cm}^{-1}$ ): 3209, 3045, 2977, 1669, 1621, 1231, 1196: ${ }^{1} \mathrm{H}-\mathrm{NMR}$ $\left(400 \mathrm{MHz}, \mathrm{CDCl}_{3}, \mathrm{rt}\right): \delta 3.66\left(\mathrm{~d}, 8 \mathrm{~Hz} 1 \mathrm{H}, \mathrm{C}_{4}\right)$, $3.94\left(\mathrm{~s}, 3 \mathrm{H}, \mathrm{OCH}_{3}\right), 5.42\left(\mathrm{~d}, 8 \mathrm{~Hz}, 1 \mathrm{H}, \mathrm{C}_{5}\right), 6.88-$ $8.03(\mathrm{~m}, 14 \mathrm{H}, \mathrm{ArH}), 9.00(\mathrm{~s}, 1 \mathrm{H}, \mathrm{NH}),{ }^{13} \mathrm{C}-\mathrm{NMR}$ $\left(100 \mathrm{MHz}, \mathrm{CDCl}_{3}, \mathrm{rt}\right): 45.12,55.85,85.7,108.22$, $114.45,121.40125 .40,128.04,129.20,129.51,130.14$, 132.24, 145.62, 150.87, 154.80, 157.20, 198.90. Anal. Calcd. for $\mathrm{C}_{23} \mathrm{H}_{19} \mathrm{NO}_{3}$ : C 77.29, H 5.36, N 3.92 , found C 77.40, H 5.16, N 3.90. LC-MS m/z: 358.14 $(\mathrm{M}+1)^{+}$. 
2.4n Isoxazoline 3eb: Obtained as yellow oil from chalcone 1e and oxime $\mathbf{2 b}$, IR (neat, $\mathrm{cm}^{-1}$ ): 3186, 3052, 2981, 1680, 1623, 1231, 1196: ${ }^{1} \mathrm{H}-\mathrm{NMR}(400 \mathrm{MHz}$, $\left.\mathrm{CDCl}_{3}, \mathrm{rt}\right): \delta 3.53\left(\mathrm{~d}, 8.4 \mathrm{~Hz}, 1 \mathrm{H}, \mathrm{C}_{4}\right), 3.86(\mathrm{~s}, 6 \mathrm{H}$, $\left.\mathrm{OCH}_{3}\right), 5.30\left(\mathrm{~d}, 8.4 \mathrm{~Hz}, 1 \mathrm{H}, \mathrm{C}_{5}\right), 6.80-8.07(\mathrm{~m}, 13 \mathrm{H}$, $\mathrm{ArH}), 9.10$ (br, $1 \mathrm{H}, \mathrm{NH}) .{ }^{13} \mathrm{C}-\mathrm{NMR}\left(100 \mathrm{MHz}, \mathrm{CDCl}_{3}\right.$, rt): 43.92, 57.15, 81.76, 104.01, 114.67, 121.87, 125.09, $128.01,128.37,128.82,130.72,131.21,135.11$, 152.91, 155.03, 161.62, 162.91, 163.14, 198.04. Calcd. for $\mathrm{C}_{24} \mathrm{H}_{21} \mathrm{NO}_{4}$ : C74.40, H 5.46, N 3.62, found C 74.11, H 5.59, N 3.32. LC-MS m/z: $388.15(\mathrm{M}+1)^{+}$.

2.4o Isoxazoline 3ec: Obtained as brown oil from chalcone 1e and oxime 2c, IR (neat, $\mathrm{cm}^{-1}$ ): 3213, 3066, 2974, 1681, 1611, 1228, 1184: ${ }^{1} \mathrm{H}-\mathrm{NMR}(400 \mathrm{MHz}$, $\left.\mathrm{CDCl}_{3}, \mathrm{rt}\right): \delta 3.55\left(\mathrm{~d}, 8 \mathrm{~Hz}, 1 \mathrm{H}, \mathrm{C}_{4}\right), 3.89-3.97$ $\left(2 \mathrm{~s}, 9 \mathrm{H}, \mathrm{OCH}_{3}\right), 5.55\left(\mathrm{~d}, 8 \mathrm{~Hz}, 1 \mathrm{H}, \mathrm{C}_{5}\right), 6.81-8.02$ (m, 12H, ArH), 9.00 (s, 1H, NH). ${ }^{13} \mathrm{C}-\mathrm{NMR}(100 \mathrm{MHz}$, $\left.\mathrm{CDCl}_{3}, \mathrm{rt}\right): 46.49,57.20,87.13,106.72,113.55,121.78$, $125.61,127.77,128.23,131.64,134.52,138.10$, 148.13, 150.30, 155.28, 159.90, 160.76, 198.42. Anal. Calcd. for $\mathrm{C}_{25} \mathrm{H}_{23} \mathrm{NO}_{5}$ : C 71.93, $\mathrm{H}$ 5.55, N 3.36, found C 71.80, H 5.82, N 3.12. LC-MS m/z: 418.16 $(\mathrm{M}+1)^{+}$.

2.4p Isoxazoline 3fa: Obtained as brown oil from chalcone 1f and oxime 2a, IR (neat, $\mathrm{cm}^{-1}$ ): 3194, 3057 , 2982, 1673, 1625, 1288: ${ }^{1} \mathrm{H}-\mathrm{NMR}\left(400 \mathrm{MHz}, \mathrm{CDCl}_{3}\right.$, rt): $\delta 2.97\left(\mathrm{~s}, 6 \mathrm{H}, \mathrm{N}\left(\mathrm{CH}_{3}\right)_{2}\right), 3.43\left(\mathrm{~d}, 8.2 \mathrm{~Hz}, 1 \mathrm{H}, \mathrm{C}_{4}\right)$, $5.50\left(\mathrm{~d}, 8.2 \mathrm{~Hz}, 1 \mathrm{H}, \mathrm{C}_{5}\right), 7.27-8.24(\mathrm{~m}, 14 \mathrm{H}, \mathrm{ArH})$, 10.03 (s, $1 \mathrm{H}, \mathrm{NH}) .{ }^{13} \mathrm{C}-\mathrm{NMR}\left(100 \mathrm{MHz}, \mathrm{CDCl}_{3}, \mathrm{rt}\right)$ : $41.16,46.10,83.55,109.78,114.51,124.50,127.71$, $128.43,128.64,128.92,130.11,132.45,133.63$, 138.52, 149.32, 153.13, 158.50, 196.20. Anal. Calcd. for $\mathrm{C}_{24} \mathrm{H}_{22} \mathrm{~N}_{2} \mathrm{O}_{2}$ : C 77.81, H 5.99, $\mathrm{N} \mathrm{7.56,} \mathrm{found} \mathrm{C}$ 77.71, H 6.04, N 7.41. LC-MS m/z: $371.20(\mathrm{M}+1)^{+}$.

2.4q Isoxazoline $3 \mathrm{fb}$ : Obtained as yellow oil from chalcone $\mathbf{1 f}$ and oxime $\mathbf{2 b}$, IR (neat, $\mathrm{cm}^{-1}$ ): 3112,3042 , 2989, 1690, 1623, 1238, 1184: ${ }^{1} \mathrm{H}-\mathrm{NMR}(400 \mathrm{MHz}$, $\left.\mathrm{CDCl}_{3}, \mathrm{rt}\right): \delta 2.77\left(\mathrm{~s}, 6 \mathrm{H}, \mathrm{N}\left(\mathrm{CH}_{3}\right)_{2}\right), 3.30(\mathrm{~d}, 8 \mathrm{~Hz}$, $\left.1 \mathrm{H}, \mathrm{C}_{4}\right), 3.79\left(\mathrm{~s}, 3 \mathrm{H}, \mathrm{OCH}_{3}\right), 5.63(\mathrm{~d}, 8 \mathrm{~Hz}, 1 \mathrm{H}$, $\left.\mathrm{C}_{5}\right)$, 6.81-8.08 (m, 13H, ArH), $9.13(\mathrm{br}, 1 \mathrm{H}, \mathrm{NH})$. ${ }^{13} \mathrm{C}-\mathrm{NMR}\left(100 \mathrm{MHz}, \mathrm{CDCl}_{3}, \mathrm{rt}\right): 40.51,45.83,58.41$, $85.13,110.52,115.12,121.30,127.71,127.93,128.21$, $130.12,132.21,132.97,138.43,142.45,154.73$, 157.83, 164.11, 199.57. Anal. Calcd. for $\mathrm{C}_{25} \mathrm{H}_{24} \mathrm{~N}_{2} \mathrm{O}_{3}$ : C 74.98, H 6.04, N 7.00, found C 74.73, H 5.97, N 7.09. LC-MS m/z: $401.18(\mathrm{M}+1)^{+}$. 2.4r Isoxazoline $3 \mathrm{fc}$ : Obtained as yellow oil from chalcone 1f and oxime 2c, IR (neat, $\mathrm{cm}^{-1}$ ): 3200, 3062, 2970, 1688, 1619, 1239, 1183: ${ }^{1} \mathrm{H}-\mathrm{NMR}(400 \mathrm{MHz}$, $\left.\left.\mathrm{CDCl}_{3}\right), \mathrm{rt}\right): \delta 2.82\left(\mathrm{~s}, 6 \mathrm{H}, \mathrm{N}\left(\mathrm{CH}_{3}\right)_{2}\right), 3.32(\mathrm{~d}, 8.4 \mathrm{~Hz}$, $\left.1 \mathrm{H}, \mathrm{C}_{4}\right), 3.85\left(\mathrm{~s}, 6 \mathrm{H}, \mathrm{OCH}_{3}\right), 5.43(\mathrm{~d}, 8.4 \mathrm{~Hz}, 1 \mathrm{H}$, $\left.\mathrm{C}_{5}\right), 6.68-8.01(\mathrm{~m}, 12 \mathrm{H}, \operatorname{ArH}), 9.74(\mathrm{~s}, 1 \mathrm{H}, \mathrm{NH})$. ${ }^{13} \mathrm{C}-\mathrm{NMR}\left(100 \mathrm{MHz}, \mathrm{CDCl}_{3}, \mathrm{rt}\right): 42.17,45.61,55.23$, 86.64, 108.90, 112.20, 115.33, 125.84, 127.73, 128.36, $128.93,130.44,131.60,138.13,145.50,148.87$, 150.51, 154.73, 157.35, 194.78. Anal. Calcd. for $\mathrm{C}_{26} \mathrm{H}_{26} \mathrm{~N}_{2} \mathrm{O}_{4}$ : C 72.54, H 6.09, N 6.51, found C 72.44, H 6.15, N 6.44. LC-MS m/z: $431.19(\mathrm{M}+1)^{+}$.

2.4s Isoxazoline 3ga: Obtained as brown oil from chalcone 1g and oxime 2a, IR (neat, $\mathrm{cm}^{-1}$ ): 3158, 3091, 2982, 1681, 1619, 1221, 1189: ${ }^{1} \mathrm{H}-\mathrm{NMR}(400 \mathrm{MHz}$, $\left.\mathrm{CDCl}_{3}, \mathrm{rt}\right): \delta 3.46\left(\mathrm{~d}, 8.4 \mathrm{~Hz}, 1 \mathrm{H}, \mathrm{C}_{4}\right), 3.87(\mathrm{~s}$, $\left.6 \mathrm{H}, \mathrm{OCH}_{3}\right), 5.50\left(\mathrm{~d}, 8.4 \mathrm{~Hz}, 1 \mathrm{H}, \mathrm{C}_{5}\right), 6.86-8.20(\mathrm{~m}$, 13H, ArH), $10.00(\mathrm{~s}, 1 \mathrm{H}, \mathrm{NH}),{ }^{13} \mathrm{C}-\mathrm{NMR}(100 \mathrm{MHz}$, $\left.\mathrm{CDCl}_{3}, \mathrm{rt}\right)$ : 45.70, 58.17, 85.48, 109.20, 114.20, 121.25, $128.74,130.73,132.19,132.62,133.07,136.53$, 144.97, 146.82, 152.80, 156.60, 199.06. Anal. Calcd. for $\mathrm{C}_{24} \mathrm{H}_{21} \mathrm{NO}_{4}$ : C 74.40, $\mathrm{H} \mathrm{5.46,} \mathrm{N} \mathrm{3.62,} \mathrm{found} \mathrm{:} \mathrm{C}$ 74.31, H 5.77, N 3.44. LC-MS m/z: $388.15(\mathrm{M}+1)^{+}$.

2.4t Isoxazoline $3 \boldsymbol{g b}$ : Obtained as yellow oil from chalcone $\mathbf{1 g}$ and oxime 2b, IR (neat, $\mathrm{cm}^{-1}$ ): ${ }^{1} \mathrm{H}-$ NMR $\left(400 \mathrm{MHz}, \mathrm{CDCl}_{3}\right): \delta 3.42(\mathrm{~d}, 8.4 \mathrm{~Hz}, 1 \mathrm{H}$, $\left.\mathrm{C}_{4}\right), 3.79\left(\mathrm{~s}, 9 \mathrm{H}, \mathrm{OCH}_{3}\right), 5.25\left(\mathrm{~d}, 8.4 \mathrm{~Hz}, 1 \mathrm{H}, \mathrm{C}_{5}\right)$, 6.88-8.02 (m, 12H, ArH), $9.12(\mathrm{br}, 1 \mathrm{H}, \mathrm{NH}) .{ }^{13} \mathrm{C}-$ NMR $\left(100 \mathrm{~Hz}, \mathrm{CDCl}_{3}, \mathrm{rt}\right): 45.20,58.90,84.90,108.10$, 109.5, 112.60, 127.85, 128.00, 128.10, 129.16, 135.35, 136.52, 139.64, 152.30, 156.20, 159.8, 199.30. Anal. Calcd. for $\mathrm{C}_{25} \mathrm{H}_{23} \mathrm{NO}_{5}$ : C 71.93, $\mathrm{H}$ 5.55, N 3.36, found C 71.80, H 5.60, N 3.19. LC-MS m/z: 418.16 $(\mathrm{M}+1)^{+}$.

$2.4 \mathrm{u}$ Isoxazoline 3gc: Obtained as yellow oil from chalcone $1 \mathrm{~g}$ and oxime 2c, IR (neat, $\mathrm{cm}^{-1}$ ): 3210, 3061, 2974, 1680, 1611, 1235, 1197: ${ }^{1} \mathrm{H}-\mathrm{NMR}(400 \mathrm{MHz}$, $\left.\mathrm{CDCl}_{3}\right): \delta 3.28\left(\mathrm{~d}, 8 \mathrm{~Hz}, 1 \mathrm{H}, \mathrm{C}_{4}\right), 3.79\left(\mathrm{~s}, 12 \mathrm{H}, \mathrm{OCH}_{3}\right)$, $5.65\left(\mathrm{~d}, 8 \mathrm{~Hz}, 1 \mathrm{H}, \mathrm{C}_{5}\right), 6.88-8.02(\mathrm{~m}, 11 \mathrm{H}, \mathrm{ArH}), 9.02$ (br, $1 \mathrm{H}, \mathrm{NH}) .{ }^{13} \mathrm{C}-\mathrm{NMR}\left(100 \mathrm{~Hz}, \mathrm{CDCl}_{3}, \mathrm{rt}\right): 45.06$, 58.28, 84.95, 109.11, 115.78, 116.75, 125.61, 127.85, $128.77,131.85,133.49,138.10,141.16,146.35$, 147.52, 148.64, 152.40, 155.20, 199.21. Anal. Calcd. for $\mathrm{C}_{26} \mathrm{H}_{25} \mathrm{NO}_{6}$ : C 69.79, H 5.63, N 3.13, found C 69.70, H 5.90, N 3.10. LC-MS m/z: $448.17(\mathrm{M}+1)^{+}$. 


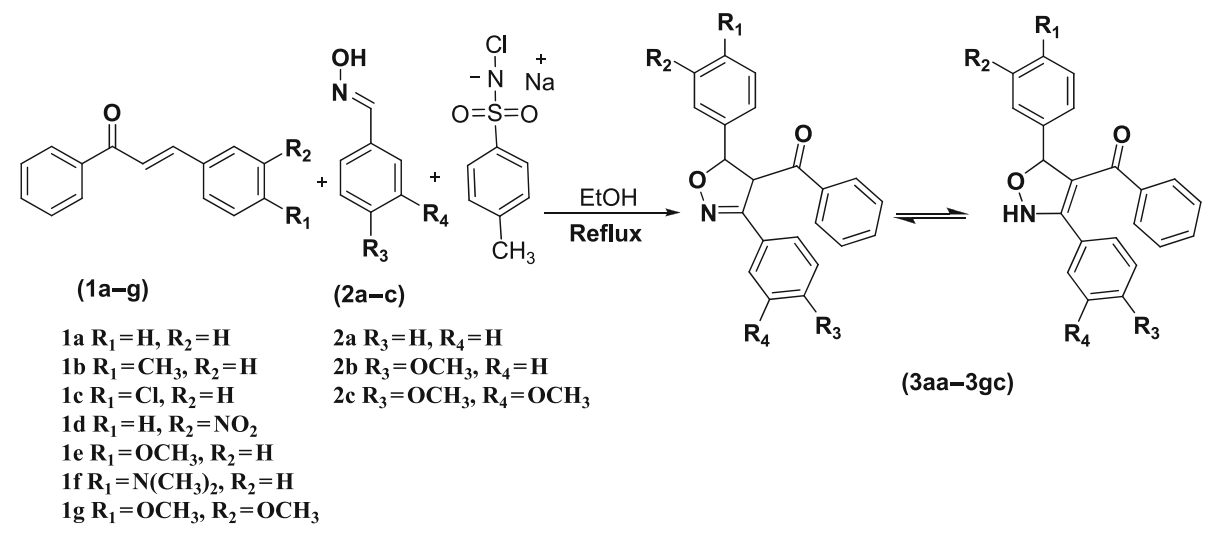

Scheme 1. General scheme for the synthesis of the 3,4,5-trisubstituted-dihydroisoxazoline derivatives.

\section{Results and discussion}

The reaction sequences for the synthesis of isoxazolines are shown in scheme 1. The desired chalcones (1a-g) were prepared in high yield by reacting the corresponding aromatic aldehydes compounds with acetophenone in the present of base. ${ }^{21}$ On the other hand the desired aldoximes $(\mathbf{2 a - c})$ were prepared by reacting the corresponding aromatic aldehyde with hydroxylamine hydrochloride in the presence of sodium acetate. ${ }^{22}$ The reaction of the chalcones $(\mathbf{1 a - g})$ with aldoximes $(\mathbf{2 a - c})$ in refluxing ethanol gave the respective isoxazolines (3aa-gc) 85-90\% yields as shown in table 1 .

On the basis of ${ }^{1} \mathrm{H}-\mathrm{NMR}$ and ${ }^{13} \mathrm{C}-\mathrm{NMR}$ spectra $\left(\mathrm{CDCl}_{3}, \mathrm{DMSO}-\mathrm{d}_{6}\right)$ the structures isolated products 3aa-gc were assigned, therefore the probable mechanism for the formation of the products involves the oxidative dehydrogenation of aromatic aldoximes by chloramine- $\mathrm{T}$ affored nitrile oxides which were intercepted in situ by chalcones in refluxing ethanol to give the products 3aa-gc. The products 3aa-gc have been isolated as apparently pure, but on basis of their spectral
${ }^{1} \mathrm{H}-\mathrm{NMR}$ and ${ }^{13} \mathrm{C}-\mathrm{NMR}\left(\mathrm{CDCl}_{3}\right)$ analyses, it is indicated there are two components exist as equilibrium of two tautomeric forms. This is because their ${ }^{1} \mathrm{H}-\mathrm{NMR}$ spectra revealed, in each case, two characteristic doublet signals near $\delta 3.32-3.74 \mathrm{ppm}$ and $\delta 5.14-5.85 \mathrm{ppm}$ which assignable to the proton of $\mathrm{C}_{4}$ and $\mathrm{C}_{5}$ groups in isoxazoline, respectively.

The ${ }^{13} \mathrm{C}-\mathrm{NMR}$ spectra at these products also in accordance with proposed structures, for example the ${ }^{13} \mathrm{C}-\mathrm{NMR}$ spectra of these compounds revealed the signals at $\delta$ 104.01-110.30 ppm, $\delta 81-88.72 \mathrm{ppm}$ and $\delta$ $152.10-155.28 \mathrm{ppm}$ assignable to the $\mathrm{C}_{4}, \mathrm{C}_{5}$ and $-\mathrm{C}=\mathrm{N}$ group of isoxazoline ring, respectively. Therefore, the ${ }^{1} \mathrm{H}-\mathrm{NMR}$ and ${ }^{13} \mathrm{C}-\mathrm{NMR}$ spectra of the products 3aagc revealed that the products most probably exist as an equilibrium of two tautomeric form (I, II) as shown in scheme 1. A tentative mechanism for the generation of nitrile oxide has been proposed in scheme 2. However, the integration of the spectra indicated that the ratio of the most tautormeric form is (50\%:50\%). The characterization of the products $\mathbf{3 a a - g c}$ by IR technique confirmed the proposed structures for example the IR

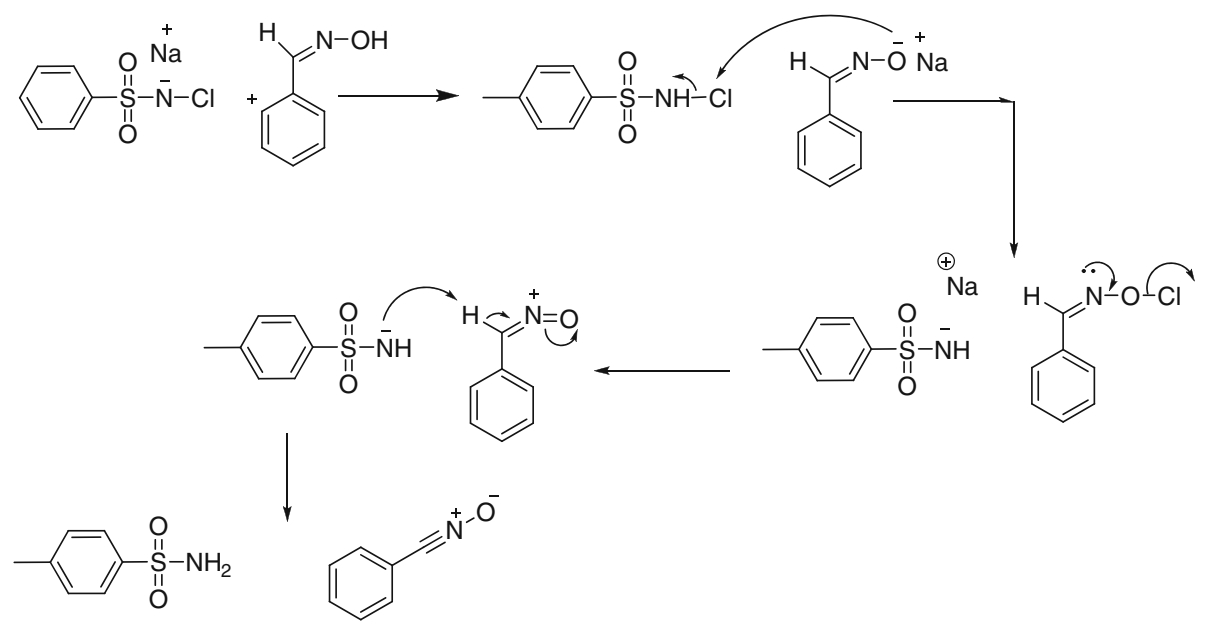

Scheme 2. Mechanism for the formation of nitrile oxides from aldoximes. 
Table 1. The structure of compounds with their yields (3aa-gc).

\begin{tabular}{|c|c|c|}
\hline Compound & Compounds & Yield (\%) \\
\hline 3aa & & 82.8 \\
\hline $3 a b$ & & 81.4 \\
\hline $3 a c$ & & 82.0 \\
\hline $3 \mathrm{ba}$ & & 82.3 \\
\hline $3 b b$ & & 81.0 \\
\hline $3 b c$ & & 80.0 \\
\hline $3 \mathrm{ca}$ & & 83.3 \\
\hline $3 \mathrm{cb}$ & & 82.0 \\
\hline $3 \mathrm{cc}$ & & 80.9 \\
\hline 3da & & 86.4 \\
\hline $3 d b$ & & 80.0 \\
\hline
\end{tabular}


Table 1. (continued).

\begin{tabular}{|c|c|c|}
\hline Compound & Compounds & Yield (\%) \\
\hline $3 d c$ & & 83.7 \\
\hline 3ea & & 84.2 \\
\hline 3eb & & 88.0 \\
\hline 3ec & & 81.7 \\
\hline $3 f a$ & & 86.9 \\
\hline $3 \mathrm{fb}$ & & 80.4 \\
\hline $3 \mathrm{fc}$ & & 84.1 \\
\hline 3ga & & 82.9 \\
\hline $3 \mathrm{gb}$ & & 81.7 \\
\hline $3 g c$ & & 89.6 \\
\hline
\end{tabular}


spectra of these compounds revealed new absorption peak at $3112-3218 \mathrm{~cm}^{-1}, 1660-1694 \mathrm{~cm}^{-1}, 1609-1628$ and $1221-1288 \mathrm{~cm}^{-1}$ due to $-\mathrm{NH}, \mathrm{C}=\mathrm{O}, \mathrm{C}=\mathrm{N}$ and $\mathrm{C}-\mathrm{O}-\mathrm{N}$ stretching frequencies, respectively. Also the IR spectra of these products revealed the aromatic $\mathrm{C}=\mathrm{C}$ and other substituent absorption at the expected regions.

\section{Conclusion}

In summary we have synthesized novel isoxazoline derivatives. The successful synthesis of new isoxazoline compounds follows a mild, efficient route with a good yield. It is known that nitrile oxides generated in situ react with alkenes and alkynes to give isoxazolines and isoxazoles, respectively. In this research work we synthesized novel isoxazolines by reacting aromatic aldoximes with chalcones ( $\alpha, \beta$-unsaturated carbonyl compounds). in the presence of chloramine-T which we wish to manipulate further.

\section{Acknowledgements}

Authors wish to thank the University of Mysore, Mysore, India, for the facilities.

\section{References}

1. Ahmad G, Mishra P K, Gupta P, Yadav P P, Tiwari P, Tamrakar A K, Srivastava A K and Maurya R 2006 Bioorg. Med. Chem. Lett. 162139

2. Gaonkar S L, Rai L K M and Prabhuswamy B 2007 Med. Chem. Res. 15407

3. Kai H, Matsumoto H, Hattori N, Takase A, Fujiwara T and Sugimoto H 2001 Bioorg. Med. Chem. Lett. 111997

4. Basappa M, Sadashiva P, Mantelingu K, Nanjunda S S and Rangappa K S 2003 Bioorg. Med. Chem. 11 4539
5. Sielecki T M, Liu J, Mousa S A, Racanelli A L, Hausner E A, Wexler R R and Olson R E 2001 Bioorg. Med. Chem. Lett. 112201

6. Ichiba T, Scheuer P J and Borges K M $1993 \mathrm{~J}$. Org. Chem. $\mathbf{5 8} 4149$

7. Srivastava S, Bajpai L K, Batra S, Bhaduri A P, Maikhuri J P and Gupta G 1999 Bioorg. Med. Chem. 72607

8. Habeeb A G, Rao P N P and Knaus E E $2001 \mathrm{~J}$. Med. Chem. 442921

9. Conti P, Dallanoce C, Amici M D, Micheli C D and Klotz K N 1998 Bioorg. Med. Chem. 6401

10. Antczak C, Bauvois B, Monneret C and Florent 2001 Bioorg. Med. Chem. 92843

11. Maurya R, Ahmad A, Gupta P, Chand k., Kumar M, Preceti R J, Rasheed N and Palit G 2011 Med. Chem. Res. 20139

12. Sadanandam A, Rajam M V, Subhash K and Rajanarendar E 1984 Indian Bot. Report 3(1) 38

13. Caramella P, Gruenanger P 1984 In 1,3-Dipolar cycloaddition chemistry, (ed.) A Padwa, New York: Wiely Interscince

14. Dannhardt G, Kiefer W, Kramer G, Maehrlein S, Nowe U and Fiebich B 2000 Eur. J. Med. Chem. 35499

15. Umesha K B, Kumar K A and Rai K M L 2002 Synth. Соттип. 321841

16. Rai K M L, Linganna N, Hassner A and Murthy C A 1992 Org. Prep. Proc. Int. 2491

17. Keigiel J, Poplawaska M, Jozwik J, Kosior M and Jurzak J 1999 Tetrahedron 405605

18. Moriya $\mathrm{O}$, Takenaka H, Iyoda M, Urata $\mathrm{Y}$ and Endo $\mathrm{T}$ 1994 J. Chem. Soc. Perkin Trans. I 413

19. (a) Rai K M L and Hassner A 1997 Indian J. Chem. 36B 242; (b) Rai K M L and Hassner A 1997 Synth. Commun. 27 467; (c) Rai K M L and Hassner A and Rai K M L 1989 Synthesis 157

20. (a) Gaonkar S L and Rai K L M 2005 J. Heterocycl. Chem. 42 877; (b) Gaonkar S L and Rai K M L 2005 Tetrahedron Lett. 465969

21. Yongjia S, Lianbing R and Jianwei W 2008 Synth. Commmun. 38583

22. Pavia D L, Lampman G M and Kriz G S 2009 Organic Chemistry A Lab Manual, Cengage Learning India Private Limited, Delhi, India

23. Brian S F, Antony J H, Peter W G and Austin R T 2011 Vogel's textbook of practical organic chemistry, 5th ed (India: Dorling Kindersley), p. 1259 